\title{
Treatment of acute abscesses in the casualty department
}

\author{
M H SIMMS, F CURRAN, R A JOHNSON, J OATES， J-C GIVEL， R CHABLOZ, \\ J ALEXANDER-WILLIAMS
}

\begin{abstract}
In the treatment of acute pyogenic soft-tissue abscess incision, curettage, and primary suture was compared with incision and drainage alone in a randomised prospective trial. Operations were performed under antibiotic cover by casualty officers, and patients were reviewed by an independent observer in a septic dressing clinic. Altogether 114 patients were studied, of whom 54 were treated by curettage and primary suture and 60 by simple drainage. The mean healing time was 8.9 days in those treated by primary suture and 7.8 days in those treated by simple drainage $(p<0.05)$. Primary healing failed to occur in $19(35 \%)$ of the sutured wounds, but there were no other complications in either group.

It is concluded that incision and drainage alone is adequate treatment for acute soft-tissue abscess.
\end{abstract}

\section{Introduction}

Surgical treatment for acute soft-tissue abscess consists primarily of incision and drainage, often complemented by curettage of the cavity and packing to permit secondary healing by granulation. Ellis in $1951^{1}$ advocated primary suture of the curetted abscess cavity under parenteral antibiotic cover and reported a high rate of primary healing of anorectal abscesses with this method. Subsequently a comparison of primary suture with the packing technique for anorectal abscess was reported from the same centre" and showed more rapid healing and less time lost from work with primary suture, with no increased risk of recurrent

\footnotetext{
Accident and Emergency Department and Department of Surgery,

The General Hospital, Birmingham

M H SIMMS, FRCS, clinical lecturer in surgery

F CURRAN, MB, CHB, senior house officer, surgery

R A JOHNSON, $\mathrm{MB}, \mathrm{CHB}$, senior house officer, surgery

$\mathrm{J}$ OATES, $\mathrm{MB}, \mathrm{CHB}$, senior house officer, surgery

J-C GIVEL, MD, registrar attached

R CHABLOZ, MD, registrar attached

J ALEXANDER-WILLIAMS, MD, FRCS, consultant surgeon
}

abscess or fistula. Acute soft-tissue abscesses at other sites have been treated satisfactorily by primary suture ${ }^{3-5}$ and Blick et al ${ }^{6}$ showed that a single injection of clindamycin one hour preoperatively is as effective as a full five-day antibiotic course in preventing postoperative bacteraemia and permitting primary healing after primary suture has been used.

Subcutaneous abscesses were known and described long before the development of surgery and the introduction of antibiotics? and were usually left to discharge pus and resolve spontaneously. Although such "treatment by neglect" causes the patient unnecessary suffering, it often works, indicating that formal curettage and debridement are not a prerequisite of treatment.

The optimal treatment for acute soft-tissue abscess should combine simplicity and safety with rapid healing and a low risk of recurrence. Admission to hospital should be avoided and dressings, nursing supervision, and time off work kept to a minimum. Primary suture meets most of these requirements but, although one trial ${ }^{2}$ showed it to be superior to deroofing and packing, it has not been shown to be superior to the simpler operation of incision and drainage alone. We therefore devised a randomised prospective trial to compare the two procedures.

\section{Patients and methods}

All patients presenting to the casualty department with an acute soft-tissue abscess from November 1980 to September 1981 inclusive were admitted to the trial, provided that the swelling was of less than 10 days' duration and had not already discharged pus. Each patient was seen by the duty surgical registrar, who confirmed the diagnosis and decided whether drainage was required. Information was recorded on standard data sheets, including details of prior antibiotic treatment and the history and physical characteristics of the abscess. The size of the abscess was the maximum diameter of subcutaneous induration, and the site was broadly classed as "anogenital" (including perineal, pilonidal, and anorectal abscesses) or "other" (including abscesses of the axilla, breast, and neck). A pilonidal abscess is an abscess in the natal cleft arising at the classical site of a pilonidal sinus, and in draining this type of abscess to relieve acute discomfort we did not aim to define or eradicate any underlying sinus. Abscesses of the hand or fingers were not included in the study.

Randomisation to treatment by either primary suture or simple drainage depended on whether the patient's casualty registration number was odd or even. All patients were given a single dose of 
$300 \mathrm{mg}$ clindamycin preoperatively, either intramuscularly one hour before operation or intravenously immediately before. No further antibiotic treatment was given. The operations were performed under general anaesthesia by junior medical staff of all grades of experience according to a written protocol.

In both treatment groups the abscess was drained through a linear incision equal to its maximum diameter and, after a pus swab had been obtained for culture, the cavity was gently explored with a finger or sinus forceps to break down loculi and promote free drainage. No further procedure was performed in patients treated by simple drainage, the wound being covered by a dry dressing. In patients treated by primary suture the cavity was then thoroughly curetted to remove granulation tissue and debris including the abscess wall and then closed by a few encircling interrupted monofilament sutures. Packs, wicks, or drains were prohibited. Immediately after each operation a blood culture was obtained. Patients were allowed home on recovery from anaesthesia, usually after two hours, and were followed up in a special septic drainage clinic at intervals of one to three days. At this clinic the wounds were inspected by one of us who had not participated in the initial surgery and the daily number of dressing changes noted. Wound healing was said to be established when tissue induration had resolved, discharge had stopped, and the scar was epithelialised. Sutures were removed on the fifth to seventh postoperative days, the interval depending on the day of first treatment, but if purulent discharge from the wound was noted sutures were removed prematurely to permit free drainage.

Healing times were compared statistically by calculating the ratio of the standard error to the difference between the means of the two groups and referring to a modification of Armitage's table of probability. ${ }^{8}$

\section{Results}

Altogether 129 patients were admitted to the trial from November 1980 to September 1981, but 15 were excluded from analysis because of deviations from the trial protocol: one was treated by cruciate incision and packing, four with chronic indurated abscesses were wrongly included, and 10 failed to return for follow-up. Of the 114 patients remaining, 54 were treated by primary suture and 60 by simple drainage. These groups were well matched for age, sex, and size of abscess (table I). Table II shows the sites of the abscesses.

TABLE I-Comparability of patients treated by primary suture and by simple drainage

\begin{tabular}{lcc}
\hline & Primary suture & Simple drainage \\
\hline No of patients & 54 & 60 \\
Male:female & $36: 18$ & $39: 21$ \\
Age (years): & $12-61$ & $8-68$ \\
Range & $31 \cdot 8$ & $30 \cdot 7$ \\
Mean & $1 \cdot 5-10$ & $1-12$ \\
Absess diameter $(\mathrm{cm}):$ & $4 \cdot 45$ & $4 \cdot 37$ \\
Range & $2 \cdot 03$ & $1 \cdot 97$ \\
Mean & & \\
SD & & \\
\hline
\end{tabular}

TABLE II-Sites of abscesses and distribution between treatment groups

\begin{tabular}{lcc}
\hline & Primary suture & Simple drainage \\
\hline Anorectal & 11 & 12 \\
Pilonidal & 5 & 9 \\
Perineal & 1 & 1 \\
\hline \multicolumn{1}{c}{ Total "anogenital" } & 17 & 22 \\
\hline Head and neck & 11 & 10 \\
Axilla & 13 & 14 \\
Breast & 3 & 2 \\
Trunk/limb & 10 & 12 \\
\hline Total "others" & 37 & 38 \\
\hline
\end{tabular}

Twenty-six of the patients treated by primary suture and 31 of those treated by simple drainage were taking antibiotics at the time of presentation. Swab cultures showed a preponderance of anaerobic and mixed infections in anogenital abscesses and of Gram-positive infections in other abscesses (table III). Resistance to clindamycing was seen only in cultures of coliform bacteria and in three anaerobic cultures (two Peptostreptococcus, one Bacteroides). All the Grampositive cultures and most anaerobic cultures were sensitive to clindamycin. Six of the seven swabs that produced no growth were from patients who were taking oral antibiotics at the time of operation. Only five positive blood cultures were obtained, of which one was $\bar{\Xi}$ probably a contaminant (Staphylococcus albus in one of three culture bottles, Staph aureus in pus swab) while the remainder duplicated thes swab culture (one Proteus, one Bacteroides, one Peptostreptococcus, and TI one Escherichia coli). Two of the positive blood cultures occurred in. patients treated by primary suture and three in patients treated by simple drainage. No patients exhibited signs or symptoms of septi- $\stackrel{\oplus}{+}$ caemia, and no postoperative antibotics were required.

TABLE III-Results of swab cultures in 92 acute soft-tissue abscesses*

\begin{tabular}{|c|c|c|c|c|c|}
\hline & \multicolumn{2}{|c|}{ Anogenital } & \multicolumn{2}{|c|}{ Others } & Total \\
\hline \multirow{7}{*}{$\begin{array}{l}\text { Sterile } \\
\text { Grampositive aerobe: } \\
\text { Staphylococcus aurelis } \\
\text { Staphylococcus albus } \\
\text { Haemolytic streptococci } \\
\text { Gram-positive aerobe anaerobe mixed } \\
\text { Anaerobe alone: } \\
\text { Peptostreptococcus Bacteroides } \\
\text { Anaerobe/coliform mixed } \\
\text { Coliform }\end{array}$} & \multicolumn{2}{|l|}{3} & \multicolumn{2}{|l|}{4} & \multirow[t]{2}{*}{7} \\
\hline & $3)$ & \multirow{3}{*}{\} $25 \cdot 8^{\prime \prime}$} & \multicolumn{2}{|l|}{$29)$} & \\
\hline & $3\}$ & & 7 & $62 \cdot 3^{\circ} \mathrm{o}$ & \multirow[t]{2}{*}{46} \\
\hline & $2\}$ & & $\begin{array}{l}2 \\
8\end{array}$ & \multirow{4}{*}{$31 \cdot 1{ }^{\prime}$} & \\
\hline & 7 & \multirow{3}{*}{$64.5{ }^{\circ}$} & 9 & & 16 \\
\hline & 5 & & & & 5 \\
\hline & 1] & & 2 & & 3 \\
\hline Total & 31 & & 61 & & 92 \\
\hline
\end{tabular}

* Cultures were not obtained in eight anogenital abscess and 14 others. Most patients required only once-daily changing of their wounde attend the clinic. Seven patients treated by primary suture and nine treated by simple drainage required a maximum of two dressings a day, and one in each group required a maximum of three dressings aco day. The mean number of return visits was 3.7 by patients treated by primary suture and 3.4 by patients treated by simple drainage.

The time in days to wound healing was compared between the twoo treatment groups both overall and with the groups subdivided by abscess site (anogenital or other) and size $(<5 \mathrm{~cm}$ or $\quad 5 \mathrm{~cm})$. Overall a. slight but significant advantage was shown for patients treated byo simple drainage (mean healing time 8.9 days in patients treated by尺 primary suture and 7.8 days in those treated by simple drainage $(\mathrm{p}<0.05)$ ), but this was not evident within the four subgroups except윽 for other abscesses $<5 \mathrm{~cm}$ (table IV). In 19 patients treated by primary

TABLE IV-Time taken by abscesses to heal

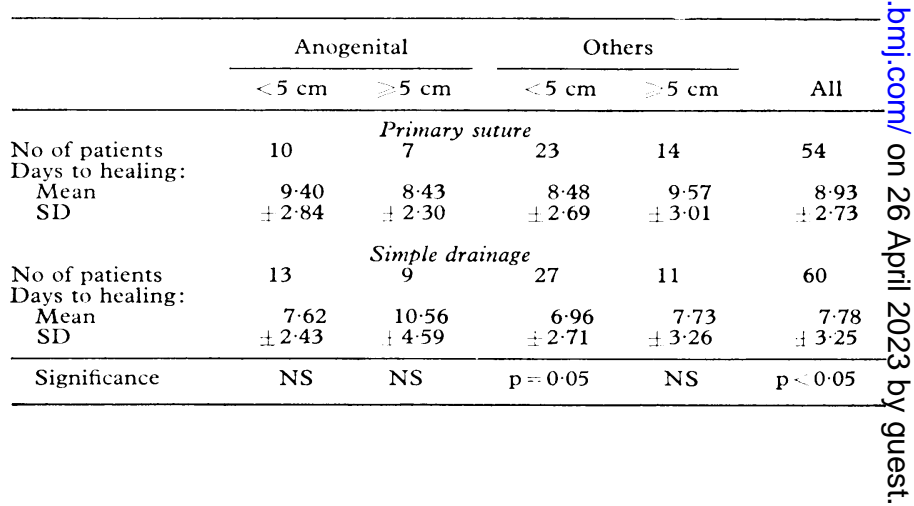

suture $(35 \%)$ pus continued to discharge from the wound postoperatively and sutures were removed prematurely to preventō reloculation. This caused the healing time to be slightly prolonged (mean $10 \cdot 6$ days), but a second drainage procedure was required in only one patient. Failure of primary healing was not associated with any particular site of abscess. In patients treated by simple drainage no additional drainage procedures were required. Three patients subsequently returned at intervals of four to six months with recurrento symptoms requiring another operation-namely, one patient treated by simple drainage with recurrent perianal abscess and one patient from each treatment group with persistent pilonidal sinus. 


\section{Discussion}

The natural history of acute soft-tissue abscess is characterised by rapid resolution after drainage of pus. Although surgical intervention precipitates drainage, procedures that entail excision of tissue or tamponade by packing may delay subsequent healing. Ellis ${ }^{9}$ taught that the abscess wall prevented access of blood-borne antibiotics to the abscess cavity and that if this wall was curetted away the cavity could fill with antibiotic-laden blood clot, permitting safe primary closure. After drainage of pus alone, however, there is usually a rapid diminution in the size of the acute abscess cavity, which may in part be due to proliferation of the abscess wall. Our results suggest that curettage of acute abscess cavities is unnecessary providing the skin wound is left open. This does not apply to carbuncles, infected cysts, and long-standing abscesses in which tissue fibrosis may delay healing.

The mean healing time for sutured abscesses in this study ( 8.9 days) is slower than the 6.6 days reported by Jones and Wilson in $1976 .{ }^{3}$ This discrepancy may reflect the essentially subjective nature of clinical estimates of healing; direct comparison of healing times in separate studies is of doubtful validity. By the same token our study, like others previously reported, did not exclude possible observer bias.

The role of antibiotics in the surgery of acute abscess is controversial. Rutherford et $a l^{10}$ found no improvement in healing rates with cluxacillin cover in a placebo-controlled trial of abscesses mostly treated by incision, curettage, and primary closure. In similar cases Blick et $a l^{6}$ showed more rapid healing with avoidance of bacteraemia when patients were given parenteral clindamycin before operation. We thought that patients undergoing curettage of an abscess should be protected from septicaemia by adequate circulating concentrations of an effective antibiotic at the time of operation. As we were comparing two techniques rather than studying the influence of antibiotics we gave clindamycin to both treatment groups. The risk of pseudomembranous colitis developing after short courses or single doses of clindamycin is extremely remote in the experience of the Leeds group, ${ }^{3}$ and this antibiotic covers most relevant organisms, as we have confirmed. Although we do not know whether systemic antibiotic cover confers any advantage when simple drainage is used, Ritchie ${ }^{11}$ showed that introducing fusidic acid (Fucidin) gel into the cavity improves the healing rate of curetted unsutured abscesses.
We deliberately delegated operations to relatively inexperienced junior staff, thus reflecting normal NHS practice in the management of minor sepsis in casualty departments. Whatever surgical procedure is advocated in this context should not strain the competence of the newly qualified or inexperienced doctor.

From this study we conclude that incision and curettage with primary suture and simple drainage alone are equally effective surgical treatments for acute soft-tissue abscesses when covered by a systemic antibiotic. Because simple drainage consumes less time and fewer materials than primary suture we now recommend it as the preferred treatment in our casualty department.

We are indebted to the 26 members of the hospital junior staff who performed the operations and to Sisters J Blundell and J Rowley and their fellow nurses who supervised the care of patients in the operating theatre and the dressing clinic. We thank Miss P Cole for preparing and typing the manuscript.

\section{References}

${ }^{1}$ Ellis M. Use of penicillin and sulphonamides in the treatment of suppuration. Lancet $1951 ; \mathrm{i}: 774-5$.

${ }^{2}$ Leaper JJ, Page RE, Rosenberg IL, Wilson DH, Goligher JL. A controlled study comparing the conventional treatment of idiopathic anorecta abscess with that of incision, curettage and primary suture under systemic antibiotic cover. Dis Colon Rectum 1976;19:46-50.

${ }^{3}$ Jones NAG, Wilson DH. The treatment of acute abscesses by incision, curettage and primary suture and antibiotic cover. Br $\mathcal{F}$ Surg 1976;63: 499-501.

${ }^{4}$ Benson EA, Goodman MA. Incision with primary suture in the treatment of acute puerperal breast abscess. Br F Surg 1970;57:55-8.

5 Page RE. Treatment of axillary abscesses by incision and primary suture under antibiotic cover. Br $\mathcal{F}$ Surg 1974;61:493-4.

${ }^{6}$ Blick PWH, Flower MW, Marsden AK, Wilson DH, Ghaneim ATM. Antibiotics in surgical treatment of acute abscesses. Br Med F 1980;281 : 111-2.

7 Rosner P, trans. Fulius Preuss' biblical and talmudic medicine. New York: Sanhedrin Press, 1978:194.

${ }^{8}$ Swinscow TDV. Statistics at square one. 7th ed. London: BMA, 1930:26.

${ }^{9}$ Ellis M. The casualty officers handbook. Vol 3. London: Butterworth, 1970 : 159-64.

${ }^{10}$ Rutherford WH, Calderwood JW, Hart D, Merrett JD. Antibiotics in surgical treatment of septic lesions. Lancet 1970;i:1077-80.

${ }^{11}$ Ritchie IC. Technique to improve the rate of healing of incised abscesses. Br Med F 1972;ii:381-3.

(Accepted 16 April 1982)
onIONS are so well known that I need not spend time about writing a description of them.

Mars owns them, and they have gotten this quality, to draw any corruption to them, for if you peel one, and lay it upon a dunghill, you shall find it rotten in half a day, by drawing putrefaction to it; then, being bruised and applied to a plague sore, it is very probable it will do the like. Onions are flatulent, or windy ; yet they do somewhat provoke appetite, increase thirst, ease the belly and bowels, provoke women's courses, help the biting of a mad dog, and of other venomous creatures, to be used with honey and rue, increase sperm, especially the seed of them. They also kill worms in children if they drink the water fasting wherein they have been steeped all night. Being roasted under the embers, and eaten with honey or sugar and oil, they much conduce to help an inveterate cough, and expectorate the cough phlegm. The juice being snuffed up into the nostrils, purges the head, and helps the lethargy (yet the often eating them is said to procure pains in the head). It hath been held by divers country people a great preservative against infection, to eat Onions fasting with bread and salt: As also to make a great Onion hollow, filling the place with good treacle, and after to roast it well under the embers, which, after taking away the outermost skin thereof, being beaten together, is a sovereign salve for either plague or sore, or any other putrefied ulcer. The juice of Onions is good for either scalding or burning by fire, water, or gunpowder, and used with vinegar, takes away all blemishes, spots and marks in the skin: and dropped in the ears, eases the pains and noise of them. Applied also with figs beaten together, helps to ripen and break imposthumes, and other sores.

Leeks are as like them in quality, as the pome-water is like an apple: They are a remedy against a surfeit of mushrooms, being baked under the embers and taken, and being boiled and applied very warm, help the piles. In other things they have the same property as the Onions, although not so effectual. (Nicholas Culpeper (1616-54) The Complete Herbal, 1850.)

SAVINE. To describe a plant so well known is needless, it being nursed up almost in every garden, and abides green all the Winter.

It is under the dominion of Mars, being hot and dry in the third degree, and being of exceeding clean parts, is of a very digesting quality. If you dry the herb into powder, and mix it with honey, it is an excellent remedy to cleanse old filthy ulcers and fistulas; but it hinders them from healing. The same is excellently good to break carbuncles and plague-sores; also helps the king's evil, being applied to the place. Being spread over a piece of leather, and applied to the navel, kills the worms in the belly, helps scabs and itch, running sores, cankers, tetters, and ringworms; and being applied to the place, may haply cure venereal sores. This I thought good to speak of, as it may be safely used outwardly, for inwardly it cannot be taken without manifest danger. (Nicholas Culpeper (161i-54) The Complete Herbal, 1850.) 